\title{
Construyendo conceptos
}

sobre electricidad en infantil

mediante actividades de indagación

\section{Constructing concepts about electricity in early childhood education through inquiry-based activities}

\author{
Nuria Calo Mosquera, Isabel García-Rodeja Gayoso, Vanessa Sesto Varela \\ Departamento de Didácticas Aplicadas, Universidade de Santiago de Compostela, Santiago de Compostela, A Coruña, España \\ nuriacalotrazo@gmail.com, isabel.garcia-rodeja@usc.es, vanessa.sesto@usc.es
}

RESUMEN - Se presenta un estudio que tiene por objetivo describir la implementación de una propuesta donde niños de educación infantil construyen conceptos y generalizaciones a través de la experiencia empírica y el diálogo con otros al implicarse en actividades de indagación con circuitos eléctricos. Los participantes son niños ( 4 a 5 años) de una clase de educación infantil. Las sesiones fueron grabadas y analizadas. Los resultados muestran que los niños entienden la electricidad como un ente que permite que las cosas funcionen. En general, no tienen dificultad para identificar aparatos eléctricos y electrónicos. A medida que se implementa la actividad los niños llegan a la generalización inductiva de que los objetos de metal conducen la electricidad. Se observa que los niños muestran más dificultad en aplicar esta idea para hacer predicciones que para justificar las observaciones realizadas.

PALABRAS CLAVE: Circuitos eléctricos; Actividades de indagación; Educación infantil; Estudio de caso.

ABSTRACT - We present a study that aims to describe the implementation of a proposal where preschool children construct concepts and generalizations through empirical experience and dialogue with others by engaging in inquiry activities on electrical circuits. The participants are children (aged 4 to 5) from a preschool classroom. The sessions were recorded and analysed. The results show that children understand electricity as an entity that allows things to work. In general, they have no difficulty identifying electrical and electronic devices. As the activity develops, children reach an inductive generalization according to which metal objects conduct electricity. It is observed that children show more difficulties in applying these ideas when they make predictions than when they justify their observations.

KEYWORDS: Electrical circuits; Inquiry-based activities; Early childhood education; Case study.

Recepción: febrero 2020 • Aceptación: septiembre 2020 • Publicación: junio 2021 


\section{INTRODUCCIÓN}

En los últimos años está creciendo el interés por la enseñanza de las ciencias en educación infantil (French, 2004). Cada vez hay un mayor reconocimiento de que la ciencia puede ser un dominio particularmente importante en la primera infancia, y que tiene un gran potencial no solo para construir una base para la comprensión científica futura, sino también para desarrollar habilidades importantes y actitudes positivas hacia el aprendizaje (Worth, 2010).

Eshach y Fried (2005) analizaron diferentes razones para introducir a los niños de educación infantil en entornos donde incluir la educación científica y señalan, entre otras razones, la curiosidad innata que sienten los niños hacia las cosas de las que trata la ciencia y que ayuda al desarrollo de las habilidades cognitivas de los niños. Además, hacen referencia a la importancia de desarrollar actitudes positivas hacia la ciencia desde edades tempranas y señalan el interés de desarrollar actividades que ayuden a los niños a generar referentes, como una primera aproximación a los conceptos científicos, al exponer a los niños fenómenos científicos. Por otro lado, se considera que la educación científica, al implicar a los estudiantes en actividades de indagación, contribuye al desarrollo del razonamiento científico. Al formular preguntas, interpretar datos y coordinar las evidencias con las teorías, los estudiantes desarrollan habilidades intelectuales que los capacitan para construir nuevo conocimiento (Chan, Burtis y Bereiter, 1997).

Sin embargo, introducir la ciencia en educación infantil es una tarea sumamente delicada, y requiere explorar y comprender las percepciones de los nińos, conocer los aspectos claves del contenido científico y ser capaces de diseñar intervenciones y ambientes de aprendizaje adecuados.

Varios estudios han examinado el aprendizaje científico y la enseñanza en educación infantil y han brindado posibles explicaciones sobre cómo las actitudes y las creencias de los maestros de educación infantil hacia la ciencia afectan a su comportamiento en el diseño e implementación de actividades de ciencias en estos niveles (Cantó, de Pro y Solbes, 2016; Pendergast, Lieberman-Betz y Vail, 2017). Los resultados del trabajo de Pendergast et al. (2017) en Estados Unidos, donde estudian las actitudes y creencias con respecto a la ciencia de 122 profesores de niveles de educación infantil, parecen indicar que los maestros entienden los beneficios de la ciencia para el desarrollo global de los niños pequeños. Sin embargo, señalan que los maestros continúan sintiéndose poco preparados en temas de ciencias $y$, como consecuencia, consideran que tienen poca competencia para apoyar de forma adecuada el aprendizaje científico de los niños. Cantó et al. (2016) estudiaron la percepción que tienen los futuros maestros sobre la enseñanza de las ciencias que se realiza en las aulas de infantil de segundo ciclo (3 a 5 años) en España. Los resultados de este estudio ponen de manifiesto la ausencia de actividades donde se les dé a los niños y a las niñas la oportunidad de desarrollar habilidades de indagación y razonamiento, pues señalan que la forma de trabajar habitual es con fichas. Cantó et al. (2016) consideran que se necesita, a la vista de la revisión que realizan, una mayor y urgente atención a este ámbito, tanto en la investigación como en la docencia.

El interés del presente trabajo reside en que se muestra cómo los niños de educación infantil construyen referentes científicos al implicarse en actividades de indagación. Para este estudio se seleccionó el tema de la electricidad y los circuitos eléctricos, porque ofrece oportunidades para reflexionar sobre ideas que están presentes en la vida cotidiana de los nińos. La comprensión de los estudiantes sobre los circuitos eléctricos se ha estudiado ampliamente en las últimas décadas cubriendo un amplio rango de grupos de edad desde primaria hasta el nivel universitario (Asoko, 1996; Çepni y Keleş, 2006; de Pro y Rodríguez, 2010; Osborne, Black, Smith y Meadows, 1991; Shepardson y Moje, 1994; Shipstone, 1984; Varela, Manrique y Favigres, 1988). Sin embargo, apenas se han publicado investigaciones en niños de educación infantil, aunque sí existen algunos trabajos muy relevantes como los de Fleer (1991), Glauert (2009), Sá et al. (1996), Solomonidou y Kakana (2000) o Kada y Ravanis (2016). 
En este trabajo se presenta un estudio de caso que tiene como objetivo describir la implementación de una propuesta donde niños de educación infantil construyen conceptos y generalizaciones a través de la experiencia empírica y el diálogo con otros.

Este trabajo pretende dar respuesta a las siguientes cuestiones: ¿Cuáles son las ideas de los niños sobre la electricidad? ¿'Son capaces de llegar a generalizaciones inductivas a partir de la evidencia empírica? ¿Son capaces de utilizar las ideas generadas de forma inductiva para hacer predicciones acertadas y para justificar las observaciones sobre la capacidad de conducir la electricidad de distintos objetos de diferentes materiales?

\section{METODOLOGÍA}

El enfoque metodológico de esta investigación es un estudio de caso de naturaleza descriptiva y cualitativa. Los estudios de caso son recomendables cuando se conoce poco del fenómeno que hay que analizar (Yin, 2003).

\section{Participantes y contexto}

Los participantes fueron diecisiete niños (catorce niñas y tres niños) de una misma clase de educación infantil ( 4 a 5 años). En el momento de la intervención, los escolares se encontraban cursando el segundo curso del segundo ciclo de Educación Infantil en un colegio público situado en un barrio obrero de un entorno semiurbano. La intervención fue diseńada por una maestra en formación (primera autora) y por una supervisora universitaria (segunda autora). La implementación de la intervención fue llevada a cabo por la maestra en formación. En el análisis de los datos recogidos durante la implementación participaron tanto la maestra en formación como la supervisora universitaria y una investigadora en didáctica de las ciencias (tercera autora).

\section{Descripción de la intervención diseñada}

La intervención didáctica se enmarca en una perspectiva socioconstructivista. Desde esta perspectiva, el aprendizaje se concibe como un acto cultural y social (Vygotsky, 1995) y se considera la comunicación fundamental para la creación de conocimiento. La actividad tiene lugar en un contexto dialógico donde la maestra y los niños hablan, actúan y piensan sobre los fenómenos, y donde la interacción social es uno de los principales factores en la construcción del conocimiento científico (Alexander, 2017). El papel de la maestra en estas actividades fue el de recurso y guía, ofreciendo al alumnado los materiales, las experiencias y los retos necesarios para ayudar a la construcción y reconstrucción de ideas y modelos.

\section{¿Por qué circuitos eléctricos?}

El estudio de circuitos eléctricos puede aparentar ser complejo y pocas veces se integra en las aulas de educación infantil. Sin embargo, existen propuestas como la de Sá et al. (1996) y otros trabajos (por ejemplo, Fleer, 1991; Glauert, 2009; Kada y Ravanis, 2016) que parecen poner de manifiesto la adecuación de algunas actividades sobre circuitos eléctricos en educación infantil. En la actividad diseñada se trata de que los niños amplíen y prueben sus ideas sobre la electricidad y, en concreto, sobre qué materiales son conductores y cuáles son no conductores de la electricidad. 
La demanda cognitiva de los conceptos asociados a la electricidad puede pensarse que está por encima de las capacidades cognitivas de los niños; sin embargo, la propuesta se centra más en relacionar los fenómenos con las ideas que los niños generan en sus experiencias cotidianas, en hacer generalizaciones inductivas a partir de los datos que obtienen a través de la indagación con los materiales, y en implicarse en actividades que les permita hacer predicciones, probar ideas, y sacar conclusiones de la experiencia.

\section{Secuencia de actividades}

En el diseño de la secuencia didáctica se recurre a una estrategia tipo Predicción-Observación-Explicación (White y Gunstone, 1992), en la que se anima a los niños a emitir predicciones sobre un fenómeno que luego tendrán la oportunidad de observar y contrastar experimentalmente. Los contenidos conceptuales que se trabajan en esta secuencia son los elementos de un circuito eléctrico simple, la disposición de los elementos del circuito para que funcione y materiales conductores y no conductores de la electricidad. En relación con otros tipos de contenido, se trabajan procedimientos como registrar e interpretar datos en tablas, hacer predicciones, hacer observaciones, contrastar las observaciones con las predicciones y sacar conclusiones. En relación con las actitudes, se trata de fomentar la iniciativa para actuar sobre los fenómenos.

En esta secuencia (véase tabla 1), con la intención de activar las ideas de los estudiantes de infantil sobre la electricidad, en la primera sesión se planteó una asamblea donde la maestra preguntó a los niños: ¿Qué crees que es la electricidad? ¿Qué aparatos usan electricidad? ¿De dónde viene la electricidad?, etc.

En la segunda sesión, la maestra, para presentar los componentes de un circuito eléctrico simple (pila, cables, interruptor, lámpara), mostró los componentes del circuito e hizo preguntas a los niños sobre ellos. Posteriormente, los niños manipularon los circuitos para ver cómo lograr que la bombilla se encendiese. Lo que se intentaba conseguir con esta actividad era dar la oportunidad de que los niños explorasen las partes del circuito al inducir a los niños a que indagasen por sí mismos, y ayudarlos a que nombrasen los elementos del circuito introduciendo términos nuevos si fuese necesario.

En la tercera sesión, una vez que se construyó un circuito simple, se les preguntó qué ocurriría si se interrumpe el circuito y se coloca un determinado objeto. La intención es que puedan probar ideas y orientar la actividad a la generación inductiva de ideas. Los objetos no se seleccionaron al azar, sino que se seleccionaron de modo que hubiese diferentes objetos del mismo material y objetos similares de diferente material. Con esta actividad se pretendía favorecer la construcción de referentes del concepto de conductor por parte de los escolares por medio de la abstracción de características comunes. Se trató de dar la oportunidad de que hiciesen predicciones sobre si se va a encender o no la bombilla al colocar cada uno de los objetos en el circuito eléctrico. Con ayuda de la maestra los niños cubrieron una tabla de predicción con imágenes de los diferentes objetos. Posteriormente, los niños dispusieron de circuitos eléctricos para así poder realizar las comprobaciones por sí mismos y cubrir una tabla de observación. 
Tabla 1.

Secuencia de actividades

\begin{tabular}{|c|c|c|}
\hline Sesiones & Intención educativa & Desarrollo \\
\hline \multicolumn{3}{|c|}{ Sesión 1} \\
\hline $\begin{array}{l}\text { ¿Qué sabemos } \\
\text { de la electricidad? }\end{array}$ & $\begin{array}{l}\text { Activar las ideas previas que tienen los ni- } \\
\text { ńos acerca de la electricidad. }\end{array}$ & $\begin{array}{l}\text { En asamblea la maestra planteó a los niños preguntas } \\
\text { acerca de la electricidad: ¿Qué crees que es la electricidad? } \\
\text { ¿Qué aparatos usan electricidad? ¿De dónde viene la } \\
\text { electricidad? }\end{array}$ \\
\hline \multicolumn{3}{|c|}{ Sesión 2} \\
\hline $\begin{array}{l}\text { ¿Qué sabemos } \\
\text { de los circuitos } \\
\text { eléctricos? }\end{array}$ & $\begin{array}{l}\text { Dar la oportunidad de que los niños ex- } \\
\text { ploren las partes del circuito y nombren } \\
\text { los elementos. }\end{array}$ & $\begin{array}{l}\text { La maestra presentó las partes de un circuito simple mien- } \\
\text { tras hacía preguntas a los niños sobre los distintos com- } \\
\text { ponentes. } \\
\text { Materiales: circuitos eléctricos simples formados cada } \\
\text { uno por una pila, tres cables, una lámpara y un interruptor } \\
\text { didáctico de palanca. }\end{array}$ \\
\hline Montaje & $\begin{array}{l}\text { Dar la oportunidad de que los niños } \\
\text { monten circuitos. }\end{array}$ & La maestra prestó apoyo en la manipulación del circuito. \\
\hline \multicolumn{3}{|c|}{ Sesión 3} \\
\hline $\begin{array}{l}\text { Materiales } \\
\text { conductores y no } \\
\text { conductores }\end{array}$ & $\begin{array}{l}\text { Dar la oportunidad de que los nińos ha- } \\
\text { gan predicciones, y registren sus predic- } \\
\text { ciones en una tabla y realicen compro- } \\
\text { baciones. }\end{array}$ & $\begin{array}{l}\text { La maestra animó a los niños a que hiciesen predicciones } \\
\text { sobre si se encendería la bombilla al conectar los objetos al } \\
\text { circuito y a que registrasen sus predicciones en una tabla } \\
\text { de doble entrada. } \\
\text { A continuación, se les dio la oportunidad de compro- } \\
\text { barlo experimentalmente, registrar los datos en la tabla y } \\
\text { discutir sobre los resultados y las posibles explicaciones. } \\
\text { Materiales: circuitos eléctricos simples formados cada } \\
\text { uno por una pila, tres cables y una lámpara. Una cuchara } \\
\text { de plástico, una cuchara de metal, una botella de vidrio, } \\
\text { una goma de borrar, un libro, una llave, una moneda, un } \\
\text { clip de metal, un clip de plástico, una pinza de madera, un } \\
\text { collar de plata, una regla y un lápiz. }\end{array}$ \\
\hline Asamblea & $\begin{array}{l}\text { Dar la oportunidad a los niños de que } \\
\text { expliciten lo aprendido. }\end{array}$ & $\begin{array}{l}\text { La maestra realiza preguntas para comprobar qué apren- } \\
\text { dieron los nińos durante la propuesta. }\end{array}$ \\
\hline
\end{tabular}

La actividad final consistió en una asamblea en la que se hicieron preguntas que permitieron conocer en qué medida las actividades provocaron cambios en las ideas de los niños sobre la electricidad y los circuitos eléctricos simples. Además, se trató de ver si eran capaces de realizar generalizaciones inductivas al relacionar el material del que estaban constituidos los objetos con la conductividad de la electricidad. 


\section{Instrumentos de recogida y análisis de datos}

En el estudio se recogió información, a modo de diario de aula, de las observaciones de cada sesión anotando el desarrollo de las intervenciones y las manifestaciones más significativas de los niños. Además, todas las sesiones fueron grabadas en audio y vídeo, y posteriormente transcritas. Las transcripciones son literales para conservar la esencia del discurso. Con el fin de garantizar el anonimato de los nińos, los nombres reales fueron reemplazados por pseudónimos.

Para dar fiabilidad y validez a estudios interpretativos como el que se presenta en este artículo se ha recurrido a una triangulación tanto de datos como de investigadores (Moreira, 2002; Patton, 2002). La triangulación de datos hace referencia a la confrontación de diferentes fuentes de datos del estudio, en este caso la información recogida a modo de diario de aula y las grabaciones de audio y vídeo. En relación con la triangulación de investigadores, las transcripciones fueron analizadas individualmente por las autoras y posteriormente se realizó un análisis reflexivo común del análisis de todos estos registros.

\section{RESULTADOS Y DISCUSIÓN}

En la presentación de resultados en primer lugar se describen las actividades implementadas. Para cada actividad se adelanta lo acontecido en cada sesión y se muestran, a través de fragmentos de las transcripciones, eventos significativos que muestran las conversaciones mantenidas entre la maestra y los niños. De forma similar a otros trabajos (Costa, 2015), las interacciones comunicativas se presentan en tres columnas, las cuales se refieren a los turnos de habla, el diálogo transcrito y el análisis de las intervenciones con el fin de describir la intencionalidad o el significado de cada intervención.

\section{Sesión 1. Ideas previas acerca de la electricidad}

En la primera actividad se observó que los niños expresaban algunas ideas sobre el significado que le dan a la electricidad. Una primera idea es que identifican la electricidad como un ente para dar luz. Además, la electricidad se entiende como un ente que permite que las cosas funcionen, como se pude observar en el siguiente evento:

\begin{tabular}{cll} 
Turno & \multicolumn{1}{c}{ Transcripción } & \multicolumn{1}{c}{ Análisis } \\
\hline 1 & $\begin{array}{l}\text { Maestra: Hoy vamos a hacer unas actividades, a ver si os gustan, } \\
\text { pero primero os voy a hacer unas preguntas. A ver quién me sabe } \\
\text { decir: ¿Qué pensáis que es la electricidad? ¿Alguno sabe? } \\
\text { (...) }\end{array}$ & \\
& Adelaida: Para las lámparas. & Relacionan la electricidad con un ente que \\
10 & Alberto: Para las luces. & permite que los dispositivos funcionen.
\end{tabular}

En el siguiente evento se muestra cómo algunos ya identifican aparatos eléctricos y electrónicos. En concreto nombraron la televisión, las lámparas, las luces, el ordenador, las bombillas, la tableta y los móviles. 


\begin{tabular}{|c|c|c|}
\hline Turno & Transcripción & Análisis \\
\hline 17 & $\begin{array}{l}\text { Maestra: ¿Sabéis de algún aparato que use electricidad? } \\
(\ldots)\end{array}$ & Pide a los niños que mencionen aparatos eléctricos. \\
\hline 24 & Alicia: La lámpara. & Identifican aparatos eléctricos. \\
\hline 25 & $\begin{array}{l}\text { Anastasia: Las luces. } \\
(\ldots)\end{array}$ & \\
\hline 27 & Amalia: Ordenador. & Identifica el ordenador como un aparato eléctrico. \\
\hline
\end{tabular}

En los siguientes eventos la profesora pregunta a los niños si saben de dónde viene la electricidad. Algunos niños relacionan la electricidad con los truenos. Una niña, a partir de una intervención de la maestra, identifica los molinos de viento con generadores de electricidad y dice que los molinos hacen aire y hacen electricidad.

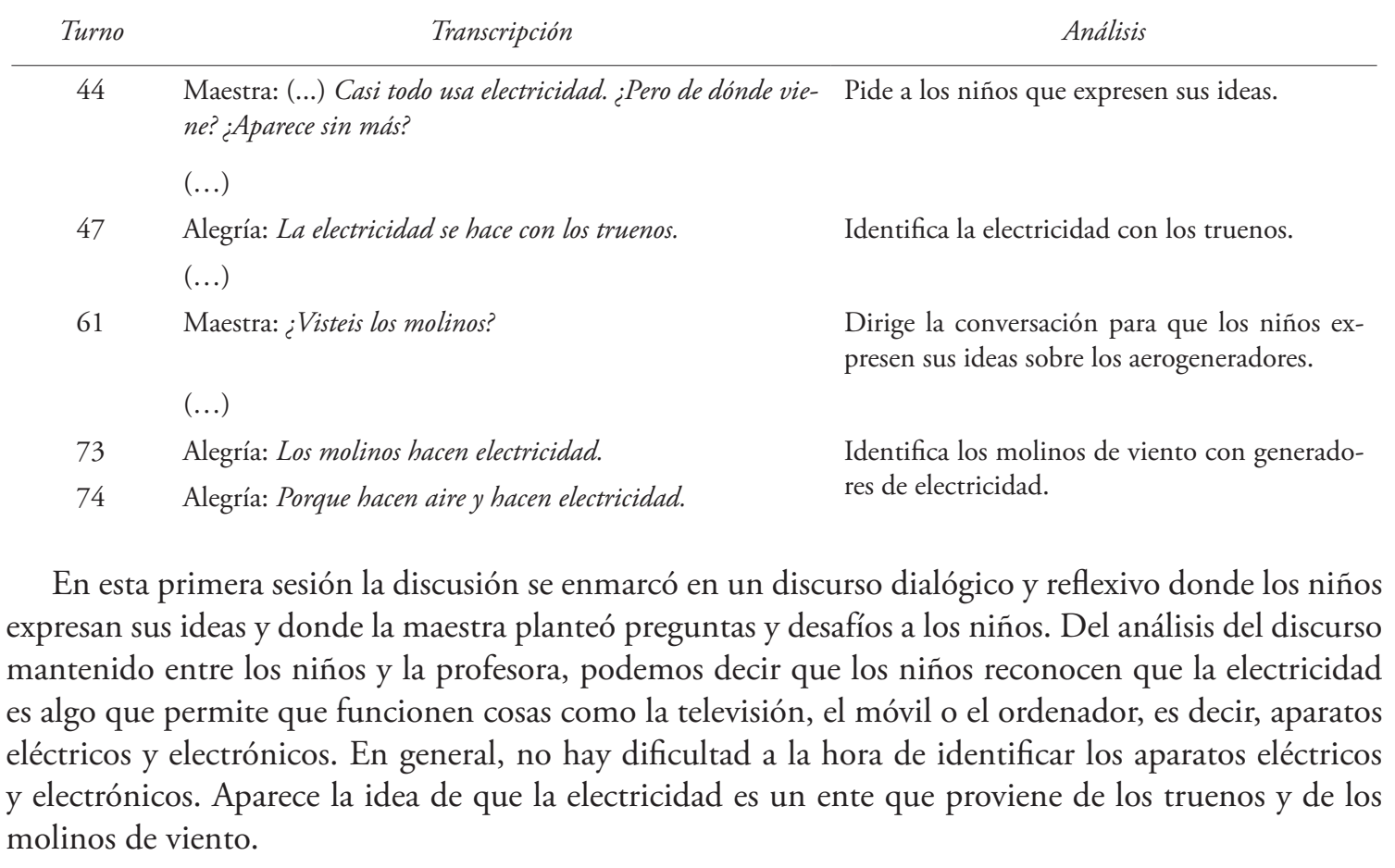

\section{Sesión 2. Circuitos}

En la siguiente sesión se hizo una pequeña asamblea para presentar los componentes de un circuito eléctrico simple: pila, cables, interruptor didáctico de palanca y lámpara. La maestra fue sacando objetos de una caja y preguntó a los niños si sabían de qué objeto se trataba. Los niños identificaron la pila, los cables y la bombilla, pero tuvieron más dificultades para identificar el interruptor. La maestra se aseguró de que sabían para qué servían los interruptores antes de ayudarlos a identificar el que iban a utilizar en el circuito.

Para continuar la actividad la maestra retó a los niños a que encendiesen una bombilla utilizando los objetos que tenía en la caja, preguntándoles qué harían para lograr que la bombilla se encendiese, de modo que les pidió que anticipasen sus acciones antes de comenzar a interactuar con los materiales. 
La maestra distribuyó a los niños en tres grupos y enseñó a los niños a enganchar los cables en los bornes de la pila de petaca y posteriormente los animó a que probasen a encender la bombilla. Algunos consiguieron encender la bombilla sin intervención de la maestra. El siguiente evento muestra la satisfacción de los niños al alcanzar el objetivo, y el comentario de Adela sugiere cómo este tipo de actividades pueden ayudar a fomentar vocaciones científicas.

\begin{tabular}{|c|c|c|}
\hline Turno & Transcripción & Análisis \\
\hline 290 & Maestra: ¿Cada cable va separado? & Pregunta por la disposición de los cables. \\
\hline 291 & $\begin{array}{l}\text { África: Podemos juntar dos. } \\
(\ldots)\end{array}$ & Realiza una propuesta. \\
\hline 293 & $\begin{array}{l}\text { Maestra: Prueba. } \\
(\ldots)\end{array}$ & Anima a la niña a probar su idea. \\
\hline 296 & $\begin{array}{l}\text { África: } \text { ¡Y se enciende la bombilla! (Aplausos) } \\
(\ldots)\end{array}$ & Muestra satisfacción al lograr encender la bombilla. \\
\hline 299 & Adela: ;Yo cuando sea mayor voy a ser cientifica! & Expresa vocación científica. \\
\hline
\end{tabular}

Los niños, emocionados, repitieron varias veces la construcción del circuito y se corrigieron unos a otros. Como vemos en el siguiente evento, ante el reto que les presentó la profesora de quitar el interruptor los niños pensaron que la bombilla se apagaría, por lo que quedaron sorprendidos al ver que no era necesario para que se encendiese la bombilla. Cuando la profesora preguntó cómo podrían apagar la bombilla sin interruptor, Alegría rápidamente desconectó el cable de la pila.

\begin{tabular}{cll} 
Turno & \multicolumn{1}{c}{ Transcripción } & \multicolumn{1}{c}{ Análisis } \\
\hline 332 & $\begin{array}{l}\text { Maestra: Una pregunta. ¿Quépasa si quitamos } \\
\text { el interruptor? }\end{array}$ & $\begin{array}{l}\text { Pregunta a los niños que sucede al quitar el inte- } \\
\text { rruptor del circuito. }\end{array}$ \\
333 & Algunas: No funciona. & $\begin{array}{l}\text { Identifica el interruptor como un elemento indis- } \\
\text { pensable para encender la bombilla. }\end{array}$ \\
334 & Maestra: ¿No funciona? ¿Queréis mirar qué & $\begin{array}{l}\text { Anima a los niños a probar lo que sucede al retirar } \\
\text { pasa si quitamos el interruptor? }\end{array}$ \\
335 & $\begin{array}{l}\text { Todos: Sí. (Desconectan el interruptor y co- } \\
\text { locan el cable en el lugar que le corresponde. }\end{array}$ & Retiran el interruptor del montaje. \\
& Gritos de sorpresa) & \\
& Todos: ;Funciona! & Muestran asombro al ver que la bombilla enciende \\
& & sin necesidad de interruptor.
\end{tabular}

En esta sesión el discurso reflexivo se representa como diálogos en los que los niños expresaron sus propias ideas, y la maestra y los niños participaron en una amplia gama de intercambios de preguntas y respuestas interactuando con el material, que ayudaron a articular ideas sobre los elementos y el funcionamiento del circuito. Como resumen de esta sesión, del análisis del discurso mantenido entre los niños y la maestra podemos decir que los niños no tuvieron dificultad en identificar los elementos básicos del circuito. Los niños fueron capaces de conectar los elementos del circuito después de varias pruebas y lograron que se encendiese la bombilla. 


\section{Sesión 3. Conductores y no conductores}

Para comenzar la sesión la profesora hizo algunas preguntas para activar las ideas de los niños sobre los circuitos eléctricos. A continuación, la maestra explicó la actividad que consistía en hacer predicciones sobre si se enciende o no la bombilla al conectar una serie de objetos al circuito formado por la pila, la lámpara y tres cables (véase figura 1). Comentó a los nińos que tenían que apuntar sus predicciones, y que tenían que comprobarlas y apuntar en otra tabla de registro las observaciones. Además, la maestra les instruyó sobre cómo debían registrar las predicciones en una tabla para pasar posteriormente a la comprobación. La profesora centró la atención de los niños en las tablas de registro y en los pictogramas. Las tablas de registro son las tablas que se utilizaron para apuntar las predicciones y las observaciones.
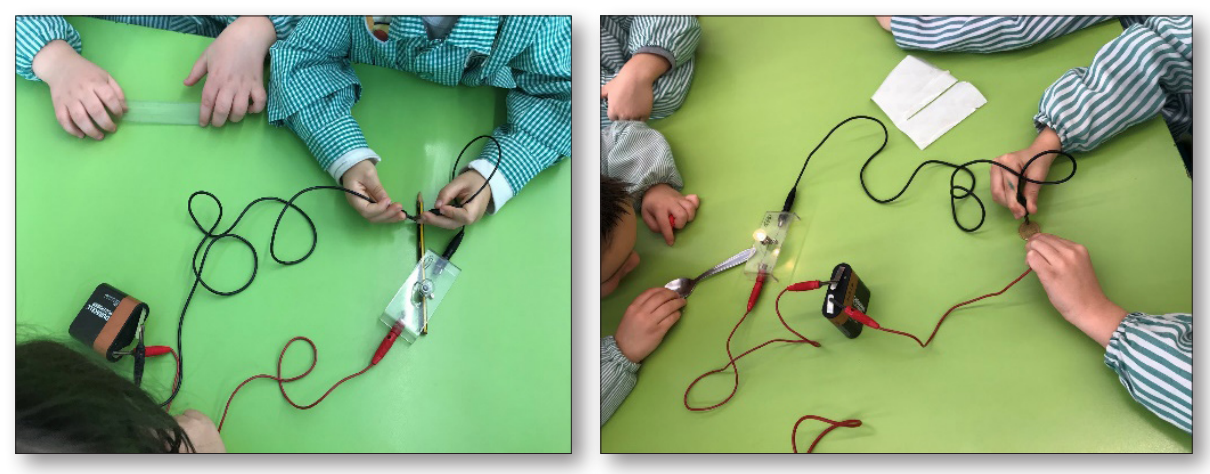

Fig. 1. Interactuando con los circuitos.

Una vez que la maestra instruyó a los niños sobre cómo cubrir la tabla de registro, les pidió que hiciesen predicciones sobre si una serie de objetos conducen o no la electricidad. Los niños fueron apuntando las predicciones en la tabla de registro y probando. El primer objeto que probaron fue una cuchara de metal y aquí no hubo acuerdo. Andrea explica que no se va a encender la bombilla porque el objeto no tiene electricidad. Como la predicción no estuvo acorde con la observación, los niños buscan otra explicación. Amalia comenta que se encendió debido a que pudo conectar con la bombilla. Aquí parece que Amalia ya está utilizando una idea de la electricidad como algo que fluye por los cables y de ahí la necesidad de conexión. Después prueban con las llaves. Otra vez no hay acuerdo en si va a encender la bombilla al conectarla a los cables del circuito. Cuando prueban observan que sí enciende la bombilla, y Amalia señala que enciende porque tiene los cables conectados.

\begin{tabular}{lll} 
Turno & \multicolumn{1}{c}{ Transcripción } & \multicolumn{1}{c}{ Análisis } \\
\hline 426 & Maestra: A ver, ¿lo intentamos? & La maestra inicia la actividad. \\
431 & Amalia: Sí. & $\begin{array}{l}\text { Predice que con la cuchara de metal sí se va a en- } \\
\text { cender la bombilla. }\end{array}$ \\
435 & Maestra: ¿Por qué creéis que va a funcionar? & Pide a los niños que justifiquen su predicción. \\
436 & $\begin{array}{l}\text { Andrea: Yo creo que no, porque la cuchara no } \\
\text { tiene electricidad. }\end{array}$ & Justifica su predicción. \\
&
\end{tabular}




\begin{tabular}{|c|c|c|}
\hline Turno & Transcripción & Análisis \\
\hline 438 & $\begin{array}{l}\text { Maestra: Que no, pues a ver, pon ahi la pe- } \\
\text { gatina. Ahora toca con esos cables la cuchara. } \\
\text { ¿Enciende la bombilla? }\end{array}$ & $\begin{array}{l}\text { Dirige la actividad para que los niños registren la } \\
\text { predicción y la comprueben. }\end{array}$ \\
\hline 439 & Todos: jSi! & Observan cómo se enciende la bombilla. \\
\hline 440 & Maestra: Fijaos, se encendió. ¿Cómo puede ser? & Pide a los niños que expliquen la observación. \\
\hline 441 & $\begin{array}{l}\text { Amalia: Porque se pudo conectar con la bom- } \\
\text { billa. } \\
(\ldots)\end{array}$ & $\begin{array}{l}\text { Muestra una idea incipiente de electricidad como } \\
\text { algo que fluye por los cables. }\end{array}$ \\
\hline 444 & $\begin{array}{l}\text { Maestra: Vamos a probar entonces. ¿Vosotros } \\
\text { pensáis que las llaves van a hacer que se en- } \\
\text { cienda la luz? }\end{array}$ & Solicita a los niños una predicción. \\
\hline 445 & $\begin{array}{l}\text { Alberto: Si. } \\
(\ldots)\end{array}$ & Predicen que con las llaves sí se va a encender. \\
\hline 451 & $\begin{array}{l}\text { África: ¡Funcionó! ¡Amalia, funcionó! ¡Con la } \\
\text { llave funcionó! }\end{array}$ & Observan cómo se enciende la bombilla. \\
\hline 452 & Maestra: ¿Y por qué? & Pide a los niños que expliquen la observación. \\
\hline 453 & Amalia: Porque tiene los cables conectados. & $\begin{array}{l}\text { Muestra una idea incipiente de electricidad como } \\
\text { algo que fluye por los cables. }\end{array}$ \\
\hline
\end{tabular}

A continuación, los nińos prueban con el libro. La mayoría cree que va a encender la bombilla, pero en un principio no encuentran una razón. África justifica la observación señalando que el objeto no es de cristal. Enseguida Anastasia hace referencia también al material del que está constituido el objeto, y señala que no enciende la bombilla porque no es de metal.

\begin{tabular}{|c|c|c|}
\hline Turno & Transcripción & Análisis \\
\hline 454 & $\begin{array}{l}\text { Maestra: ¡Muy bien! Ahora con el libro. ¿Qué } \\
\text { pensáis, que el libro va a encender la bombilla? } \\
(. . .)\end{array}$ & Solicita a los niños una predicción. \\
\hline 458 & $\begin{array}{l}\text { Andrea: Sí. } \\
(\ldots) \text {. }\end{array}$ & $\begin{array}{l}\text { Predicen que con el libro sí se va a encender la } \\
\text { bombilla. }\end{array}$ \\
\hline 460 & $\begin{array}{l}\text { Maestra: Pues ponemos que sí. ¿Por qué creéis } \\
\text { que se va a encender la bombilla? (Silencio. } \\
\text { Los niños parecen no saber qué responder) } \\
\text { (...) }\end{array}$ & Pide a los niños que justifiquen su predicción. \\
\hline 462 & $\begin{array}{l}\text { Amalia: No. } \\
(\ldots)\end{array}$ & Observa cómo no se enciende la bombilla. \\
\hline 467 & $\begin{array}{l}\text { Maestra: Vale. Pon ahi que no. ¿Y por qué } \\
\text { pensáis que no? }\end{array}$ & Pide a los niños que expliquen la observación. \\
\hline $\begin{array}{l}468 \\
469\end{array}$ & $\begin{array}{l}\text { África: Porque no es de cristal. } \\
\text { Anastasia: Ni de metal. }\end{array}$ & $\begin{array}{l}\text { Explican lo observado en base al material del que } \\
\text { está constituido el objeto. }\end{array}$ \\
\hline
\end{tabular}

Anastasia es la primera que menciona la idea de que el objeto no enciende porque no es de metal. Una vez que incorporan la idea de que no enciende la bombilla porque no es de metal utilizan esta idea para justificar sus predicciones en relación con otro objeto, la botella. Cuando la profesora le pregunta a Amalia por qué no va a encender la bombilla cuando el circuito se conecta con la botella, ella señala 
que porque no es de metal. En el caso de la moneda, África y Andrea consideran que sí va a encender la bombilla. Aurelia justifica la observación aduciendo que es de metal.

\begin{tabular}{|c|c|c|}
\hline Turno & Transcripción & Análisis \\
\hline 472 & $\begin{array}{l}\text { Maestra: (...) A ver. ¿Pensáis que la botella va } \\
\text { a encender la bombilla? }\end{array}$ & Solicita a los niños una predicción. \\
\hline 473 & $\begin{array}{l}\text { Aurelia: No. } \\
(\ldots)\end{array}$ & $\begin{array}{l}\text { Predicen que con la botella no se va a encender la } \\
\text { bombilla. }\end{array}$ \\
\hline 476 & $\begin{array}{l}\text { Amalia: } N o . \\
(\ldots)\end{array}$ & \\
\hline 478 & $\begin{array}{l}\text { Maestra: Pon entonces que no. ¿Probamos } \\
\text { ahora? }\end{array}$ & $\begin{array}{l}\text { Dirige la actividad para registrar la predicción y } \\
\text { comprobarla. }\end{array}$ \\
\hline 479 & África: No enciende. & Observa cómo no se enciende la bombilla. \\
\hline 480 & Maestra: ¿Enciende? No. ¿Por qué? & Pide a los niños que expliquen lo observado. \\
\hline \multirow[t]{2}{*}{481} & Amalia: Porque no es de metal. & $\begin{array}{l}\text { Explica lo observado en base a que el material del } \\
\text { que está constituido el objeto no es metal. }\end{array}$ \\
\hline & $(\ldots)$ & \\
\hline 484 & $\begin{array}{l}\text { Maestra: A ver, no pruebes aún. ¿Vosotros pen- } \\
\text { sáis que la moneda va a encender la bombilla? }\end{array}$ & $\begin{array}{l}\text { Solicita a los niños una predicción acerca de si con } \\
\text { una moneda se enciende la bombilla. }\end{array}$ \\
\hline 485 & $\begin{array}{l}\text { África: Si. } \\
(\ldots)\end{array}$ & $\begin{array}{l}\text { Predicen que con una moneda sí se va a encender } \\
\text { la bombilla. }\end{array}$ \\
\hline 489 & $\begin{array}{l}\text { Alberto: No. } \\
(\ldots)\end{array}$ & $\begin{array}{l}\text { Predice que con una moneda no se va a encender } \\
\text { la bombilla. }\end{array}$ \\
\hline \multirow[t]{2}{*}{491} & Amalia: Que sí. & $\begin{array}{l}\text { Predice que con una moneda sí se va a encender } \\
\text { la bombilla. }\end{array}$ \\
\hline & $(\ldots)$ & \\
\hline 493 & Amalia: ;Encendió! (Chillando). & $\begin{array}{l}\text { Observa cómo sí se enciende la bombilla al inter- } \\
\text { calar en el circuito una moneda. }\end{array}$ \\
\hline 494 & $\begin{array}{l}\text { Maestra: Encendió. Muy bien. ¿Y por qué la } \\
\text { moneda si enciende? }\end{array}$ & Pide a los nińos que expliquen lo observado. \\
\hline 495 & Aurelia: Porque es de metal. & $\begin{array}{l}\text { Explica lo observado en base a que el material del } \\
\text { que está constituido el objeto es metal. }\end{array}$ \\
\hline
\end{tabular}

A continuación, prueban con una regla de plástico. Curiosamente la predicción que hacen es que va a encender la bombilla, y es cuando ven que no se enciende la bombilla cuando Aurelia lo justifica haciendo referencia a que es de plástico. A continuación, prueban con el lápiz. No hay acuerdo en la predicción. Cuando prueban, Aurelia justifica que no enciende debido a que no es de metal.

Posteriormente prueban con otros objetos como un collar de plata, una goma y unas pinzas. En el caso del collar todos, excepto Alberto, están de acuerdo en que va a encender la bombilla. Anastasia quiere ver cómo enciende. Ahora es Anastasia quien justifica que enciende la bombilla porque el collar es de metal. 


\begin{tabular}{|c|c|c|}
\hline Turno & Transcripción & Análisis \\
\hline 521 & $\begin{array}{l}\text { Maestra: A ver. ¿Vosotros pensáis que el collar } \\
\text { va a encender la luz? } \\
\text { (...) }\end{array}$ & Solicita a los niños una predicción. \\
\hline 526 & Aurelia: Si. & $\begin{array}{l}\text { Predice que con el collar de plata sí va a encender } \\
\text { la bombilla. }\end{array}$ \\
\hline 527 & $\begin{array}{l}\text { Alberto: Yo no. } \\
(\ldots)\end{array}$ & Se opone a la predicción de su compañera. \\
\hline 529 & $\begin{array}{l}\text { África: Yo digo que sí. } \\
(\ldots)\end{array}$ & Predicen que con el collar se va a encender. \\
\hline 531 & Amalia: Yo también que sí. & \\
\hline 532 & Maestra: A ver a ver, vamos a probar. Prueba. & Pide a los niños que comprueben su predicción. \\
\hline 533 & África: ¡Enciende! ¡Mira cómo enciende! & Observan cómo se enciende la bombilla. \\
\hline 534 & $\begin{array}{l}\text { Andrea: ;Funciona! } \\
(\ldots)\end{array}$ & \\
\hline 541 & $\begin{array}{l}\text { Maestra: ¿Y por qué pensáis que enciende el } \\
\text { collar? }\end{array}$ & Pide a los niños que expliquen lo observado. \\
\hline 542 & África: Porque es de metal. & $\begin{array}{l}\text { Explica lo observado haciendo referencia a que el } \\
\text { objeto está hecho de metal. }\end{array}$ \\
\hline
\end{tabular}

En el caso del resto de los objetos no siempre aciertan en las predicciones, pero una vez que prueban y observan que no enciende la bombilla, lo justifican haciendo referencia a que no es de metal.

A continuación, ponemos un fragmento de la actividad realizada con los últimos objetos. En el siguiente evento se observa también cómo los niños fueron capaces de contrastar sus predicciones con las observaciones que habían realizado tanto en los casos en los que la observación confirma la predicción como en los casos donde esto no ocurre.

\begin{tabular}{|c|c|c|}
\hline Turno & Transcripción & Análisis \\
\hline 575 & $\begin{array}{l}\text { Maestra: Un clip. ¿Vosotros pensáis que un clip } \\
\text { va a encender la bombilla? }\end{array}$ & $\begin{array}{l}\text { Solicita a los niños una predicción acerca de si con } \\
\text { un clip de plástico se enciende la bombilla. }\end{array}$ \\
\hline 576 & $\begin{array}{l}\text { Andrea: Si. } \\
(\ldots)\end{array}$ & Predicen que se va a encender la bombilla. \\
\hline 580 & $\begin{array}{l}\text { Maestra: ¿Todas que sí? Vale, pues Aurelia } \\
\text { prueba ahi. ¿Encendió? }\end{array}$ & Pide a los niños que comprueben su predicción. \\
\hline 581 & Aurelia: No. & Observa cómo no se enciende la bombilla. \\
\hline 582 & Maestra: ¿No? ¿Y por qué? & Solicita una justificación. \\
\hline 583 & Amalia: Porque no es de metal. & $\begin{array}{l}\text { Explica lo observado haciendo referencia a que no } \\
\text { es metal. }\end{array}$ \\
\hline 584 & $\begin{array}{l}\text { Maestra: Vale. Muy bien. ¿Qué más falta? } \\
\text { ¿Vosotros pensáis que si conectamos esta cucha- } \\
\text { ra al circuito va a encender la lámpara? }\end{array}$ & $\begin{array}{l}\text { Solicita a los niños una predicción acerca de si con } \\
\text { una cuchara de plástico se enciende la bombilla. }\end{array}$ \\
\hline 585 & $\begin{array}{l}\text { Anastasia: Seguro. } \\
(\ldots)\end{array}$ & $\begin{array}{l}\text { Predice que con la cuchara de plástico sí se va a } \\
\text { encender. }\end{array}$ \\
\hline 588 & $\begin{array}{l}\text { Alberto: No. } \\
(\ldots)\end{array}$ & Se opone a las predicciones de sus compañeras. \\
\hline
\end{tabular}




\begin{tabular}{|c|c|c|}
\hline Turno & Transcripción & Análisis \\
\hline 592 & $\begin{array}{l}\text { Maestra: Vale, pues ponemos que si. A ver, eh. } \\
\text { ¿Enciende? }\end{array}$ & Pide a los niños que comprueben su predicción. \\
\hline 593 & Todos: No. & $\begin{array}{l}\text { Observan cómo no se enciende la bombilla al } \\
\text { intercalar en el circuito la cuchara de plástico. }\end{array}$ \\
\hline 594 & $\begin{array}{l}\text { Maestra: ¿Y por qué esa cuchara no encendió } \\
\text { y la otra sí? }\end{array}$ & $\begin{array}{l}\text { Pide a los nińos que expliquen lo observado con la } \\
\text { cuchara de metal y la de plástico. }\end{array}$ \\
\hline 595 & Amalia: Porque es plástico y esa, metal. & $\begin{array}{l}\text { Explica lo observado en base al material del que } \\
\text { está constituido el objeto. }\end{array}$ \\
\hline 596 & $\begin{array}{l}\text { Maestra: Muy, muy bien. Pues pon ahi, Ama- } \\
\text { lia, que esa no encendió. Solo nos queda un } \\
\text { clip. A ver. Este clip no enciende. ¿Verdad que } \\
\text { no? (Refiriéndose al clip de plástico). ¿Y este } \\
\text { entonces va a encender o no? }\end{array}$ & $\begin{array}{l}\text { Solicita a los niños una predicción acerca de si con } \\
\text { un clip de metal se enciende la bombilla. }\end{array}$ \\
\hline 597 & $\begin{array}{l}\text { Andrea: Sí. } \\
(\ldots)\end{array}$ & $\begin{array}{l}\text { Predicen que con un clip de metal sí se va a encen- } \\
\text { der la bombilla. }\end{array}$ \\
\hline 599 & $\begin{array}{l}\text { Maestra: ¿Y este por qué sí? } \\
(\ldots)\end{array}$ & Pide a los niños que se justifiquen su predicción. \\
\hline 601 & Amalia: Porque es de metal. & $\begin{array}{l}\text { Justifica la predicción en base a que el clip es de } \\
\text { metal. }\end{array}$ \\
\hline
\end{tabular}

Por último, se hizo una asamblea final para poner en común las ideas trabajadas en las actividades. Los niños recordaron las partes del circuito. La maestra les preguntó qué objetos no encendían la bombilla. Los niños señalaron el libro, la botella, la goma y un clip. Da la sensación de que los niños han sido capaces de consolidar las ideas y generalizaciones que han establecido a través de la interacción con los objetos, con los compañeros y la maestra.

\begin{tabular}{cll} 
Turno & \multicolumn{1}{c}{ Transcripción } & \multicolumn{1}{c}{ Análisis } \\
\hline 668 & $\begin{array}{l}\text { Maestra: Vale. ¿Y de esas cosas todo encendía } \\
\text { la bombilla o habia algunos objetos que no? }\end{array}$ & Pide a los niños que rememoren lo observado. \\
& $(\ldots)$ & \\
674 & Alicia: Ni el libro, ni la botella, ni la goma. & Enumera varios objetos no conductores. \\
675 & Maestra: ¿Y por qué esas cosas no encendian? & Pide a los niños que expliquen lo observado. \\
676 & Adela: Porque no eran de metal. & $\begin{array}{l}\text { Expresa la generalización de que los objetos de me- } \\
\text { tal sí encienden una bombilla. }\end{array}$
\end{tabular}

En esta sesión se observa cómo van evolucionando las ideas a través del discurso reflexivo, al hacer predicciones y al darles la oportunidad de probarlas. Al iniciar la sesión, los niños explican sus predicciones y observaciones utilizando ideas como que no enciende la bombilla porque el objeto no tiene electricidad o no está bien conectado. A medida que van probando con diversos objetos, empiezan a utilizar ideas que tienen que ver con la naturaleza del material del que está constituido el objeto. La primera intervención, en este sentido, es de África, quien indica que la bombilla no encenderá al colocar un libro en el circuito porque no es de cristal. De ahí Anastasia formula la idea de que no se enciende la bombilla porque el objeto no es de metal. En cuanto aparece esta idea la utilizan cada vez más a medida que se va confirmando en las observaciones. La idea de África desaparece del discurso una vez que se ve 
refutada por las observaciones con la botella. Llegado a este punto, en general hacen las predicciones de forma correcta con los objetos de metal como las monedas; sin embargo, fallan en la predicción en los objetos de otros materiales. Da la sensación de que algunos de los niños llegaron a la generalización inductiva de que los objetos de metal al colocarlos en el circuito permiten que se encienda la bombilla, lo que sería un referente para construir el concepto de materiales conductores de la electricidad, pero dicha generalización la utilizan con dificultad para hacer predicciones. Una vez que prueban con el objeto, y ya tienen una evidencia empírica, sí justifican la observación haciendo referencia a la naturaleza del material. En la asamblea final se ve cómo la selección de objetos similares de distintos materiales como las cucharas o los clips ayudaron a generar conflictos cognitivos cuya resolución favoreció las generalizaciones inductivas.

\section{CONCLUSIONES E IMPLICACIONES EDUCATIVAS}

En este trabajo se ha intentado describir cómo niños de 4 y 5 años y la maestra abordan el trabajo en el aula, y construyen conceptos y generalizaciones a través de la experiencia empírica y el diálogo con otros al implicarse en actividades de indagación con circuitos eléctricos.

En relación con la implementación de las sesiones, cabe señalar que estuvo enmarcada en un discurso dialógico y reflexivo donde los niños expresaron sus ideas y donde la maestra planteó preguntas y desafíos. La intervención de la maestra fue fundamentalmente para fomentar el interés, anticipar las acciones explicando lo que se pretendía con cada actividad, animar a los niños a que hiciesen predicciones y que las justificasen, y alentar a los niños a que probasen sus predicciones y que explicasen lo que observaban.

En relación con las ideas que emergen de las conversaciones de los niños cuando interaccionan entre ellos y con los fenómenos bajo estudio, hay que señalar que a lo largo de las sesiones los niños expresaron ideas diversas sobre la electricidad y ofrecieron diferentes tipos de explicaciones. Los niños reconocieron que la electricidad es algo que permite que funcionen cosas como la televisión, el móvil o el ordenador. En general, no tuvieron dificultad a la hora de identificar los aparatos eléctricos y electrónicos. Resultados similares se describen en otros trabajos sobre niños de educación infantil (Kada y Ravanis, 2016). En las conversaciones de los niños aparece la idea de que la electricidad es un ente que se genera en las tormentas y en los molinos de viento. Estas ideas se están gestando en un determinado contexto y en algunos casos son iniciadas por los niños y en otros, por la maestra; por lo tanto, tienen cierto carácter idiosincrático (Carey, 1985). Sin embargo, estas ideas están relacionadas con los hallazgos de otros autores que afirman que los niños de entre 5 y 6 años, previo a la instrucción, tienen ya ciertas representaciones sobre los fenómenos eléctricos en las que se identifica un razonamiento preenergético (Glauert, 2009; Kada y Ravanis, 2016). A diferencia de los niños de 5 a 6 años, los niños del presente estudio apenas utilizan la idea de la electricidad como algo que fluye por los cables (Kada y Ravanis, 2016). De hecho, una niña hace explícita la idea de que no se enciende la bombilla porque el objeto no tiene electricidad. Esta idea puede estar relacionada con concepciones descritas en otros trabajos donde participan niños de prescolar (Solomonidou y Kakana, 2000) y donde se describe cómo los niños perciben la electricidad como una entidad estática. Los niños dicen, por ejemplo, que la electricidad ya está en los electrodomésticos cuando los compras.

En relación con la evolución de las ideas al participar en las sesiones descritas, podemos señalar que en un principio los niños explican sus predicciones y observaciones utilizando ideas como que no enciende la bombilla porque el objeto no tiene electricidad o no está bien conectado; sin embargo, a medida que van probando con diversos objetos, empiezan a utilizar ideas que tienen que ver con la naturaleza del material del que está constituido el objeto. En cuanto se formuló la idea de que no se 
enciende la bombilla porque el objeto no es de metal, utilizan cada vez más esta idea puesto que se va confirmando en sus observaciones.

Respecto a si son capaces de llegar a generalizaciones, queremos señalar que fueron capaces de construir generalizaciones inductivas a partir de la experiencia empírica. Este resultado es similar al obtenido por Sá et al. (1996) en un estudio con niños de edades similares a los que participaron en este trabajo.

En cuanto a si son capaces de utilizar las ideas generadas de forma inductiva para hacer predicciones acertadas y para justificar las observaciones sobre la capacidad de conducir la electricidad de distintos objetos de diferentes materiales, a pesar de las limitaciones del presente trabajo, y del peligro de sacar conclusiones basadas en pocos datos, podemos apuntar que los nińos que participaron en estas actividades muestran dificultades a la hora de utilizar las ideas generadas de forma inductiva para hacer predicciones acertadas sobre la capacidad de conducir la electricidad de distintos objetos de diferentes materiales. Sin embargo, muestran una mayor competencia al utilizar las ideas generadas de forma inductiva para justificar las observaciones.

Como posibles mejoras de la propuesta apuntamos que la primera sesión debería centrarse más en cuestiones que ayuden a profundizar en el pensamiento del niño y en la segunda sesión se podría obviar el uso del interruptor haciendo la actividad más significativa. Otra cuestión mejorable es la forma de recoger los datos de las predicciones. En ese momento se debería profundizar en que justifiquen las predicciones y apuntar las predicciones de todos los niños sin que tengan que llegar a acuerdo.

Los resultados de este trabajo apoyan la importancia de realizar actividades de indagación en educación infantil para dar oportunidades para que los niños desarrollen habilidades de pensamiento y generen referentes sobre conceptos científicos. A la hora de diseñar propuestas para que los niños realicen generalizaciones inductivas, es importante realizar actividades de predicción, previas a las actividades de observación, y darles la oportunidad de que justifiquen tanto las predicciones como las observaciones. Además, se deben diseñar actividades para que utilicen las ideas generadas tanto en nuevas predicciones como en nuevas observaciones.

Se espera que este trabajo sea de utilidad para todas aquellas personas implicadas en la educación de las niñas y de los niños, y que favorezca que se implementen actividades de ciencias que ayuden al desarrollo del razonamiento, a construir referentes conceptuales y a fomentar actitudes positivas hacia la ciencia.

\section{AGRADECIMIENTOS:}

Al proyecto EDU2017-82915-R, financiado por FEDER/Ministerio de Ciencia, Innovación y Universidades - Agencia Estatal de Investigación, y al proyecto PGC2018-096581-B-C22, financiado por FEDER/Ministerio de Ciencia, Innovación y Universidades - Agencia Estatal de Investigación/ Proyecto ESPIGA.

\section{REFERENCIAS BIBLIOGRÁFICAS}

Alexander, R. (2017). Towards dialogic teaching: Rethinking classroom talk. Cambridge: Dialogos.

Asoko, H. (1996). Developing scientific concepts in the primary classroom: Teaching about electric circuits. En G. Welford, J. Osborne y P. Scott (Eds.), Research in science education in Europe: Current issues and themes (pp. 36-49). Londres: Falmer Press. 
Cantó, J., De Pro, A. y Solbes, J. (2016). ¿Qué ciencias se enseñan y cómo se hace en las aulas de educación infantil? La visión de los maestros en formación inicial. Enseñanza de las Ciencias: Revista de Investigación y Experiencias Didácticas, 34(3), 25-50.

Carey, S. (1985). Conceptual change in childhood. Londres: MIT Press.

Çepni, S. y Keleş, E. (2006). Turkish students' conceptions about the simple electric circuits. International Journal of Science and Mathematics Education, 4(2), 269-291. https://doi.org/ 10.1007/s10763-005-9001-z

Chan, C., Burtis, J. y Bereiter, C. (1997). Knowledge building as a mediator of conflict in conceptual change. Cognition and Instruction, 15(1), 1-40. https://doi.org/10.1207/s1532690xci1501_1

Costa, T. (2015). Influência da criaçâo e crítica de analogias por estudantes de química do ensino médio na promoção de interaçóes argumentativas (tesis doctoral). Ouro Preto: Universidad Federal de Ouro Preto.

De Pro Bueno, A. y Rodríguez, J. (2010). Aprender competencias en una propuesta para la enseñanza de los circuitos eléctricos en Educación Primaria. Enseñanza de las Ciencias: Revista de Investigación y Experiencias Didácticas, 28(3), 385-404.

Decreto 330/2009, de 4 de junio, por el que se establece el currículo de la educación infantil en la Comunidad Autónoma de Galicia. Diario Oficial de Galicia, 10.

Eshach, H. y Fried, M. N. (2005). Should science be taught in early childhood? Journal of Science Education and Technology, 14(3), 315-336.

https://doi.org/10.1007\%2Fs10956-005-7198-9

Fleer, M. (1991). Socially constructed learning in early childhood science education. Research in Science Education, 21(1), 96-103.

https:// doi.org/10.1007/BF02360462

French, L. (2004). Science as the centre of a coherent, integrated early childhood curriculum. Early Childhood Research Quarterly, 19, 138-149. https://doi.org/10.1016/j.ecresq.2004.01.004

Glauert, E. B. (2009). How young children understand electric circuits: Prediction, explanation and exploration. International Journal of Science Education, 31(8), 1025-1047. https://doi.org/10.1080/09500690802101950

Kada, V. y Ravanis, K. (2016). Creating a simple electric circuit with children between the ages of five and six. South African Journal of Education, 36(2), 1-9.

https://doi.org/10.15700/saje.v36n2a1233

Martínez, R. A. (2007). La investigación en la práctica educativa: Guía metodológica de investigación para el diagnóstico y evaluación en los centros docentes. Madrid: MEC.

Moreira, M. A. (2002). Investigación en educación en ciencias: Métodos cualitativos. Acatas de PIDEC, $4(14), 25-45$.

Osborne, J., Black, P., Smith, M. y Meadows, J. (1991). Primary SPACE project research report: Electricity. Liverpool: Liverpool University Press.

Patton, M. Q. (2002). Qualitative research and evaluation methods: Integrating theory and practice. Thousand Oaks: Sage Publications.

Pendergast, E., Lieberman-Betz, R. G. y Vail, C. O. (2017). Attitudes and beliefs of prekindergarten teachers toward teaching science to young children. Early Childhood Education Journal, 45(1), 43-52. https://doi.org/10.1007\%2Fs10643-015-0761-y

Sá, J., Rodrigues, A., Gomes, A., Veloso, E., Torres, G. y Silva, M. (1996). A descoberta de objectos e materiais condutores da electricidade por crianças de 4/5 anos. Aprender, 20, 65-70. 
Shepardson, D. P. y Moje, E. B. (1994). The nature of fourth graders' understandings of electric circuits. Science Education, 78(5), 489-514.

https://doi.org/10.1002/sce.3730780505

Shipstone, D. M. (1984). A study of children's understanding of electricity in simple DC circuits. European Journal of Science Education, 6(2), 185-198.

https://doi.org/10.1080/0140528840060208

Solomonidou, C. y Kakana, D. M. 2000. Preschool children's conceptions about the electric current and the functioning of electric appliances. European Early Childhood Education Research Journal, $8(1), 95-111$. https://doi.org/10.1080/13502930085208511

Varela, P., Manrique, M. J. y Favigres, A. (1988). Circuitos eléctricos: Una aplicación de un modelo de enseñanza-aprendizaje basado en las ideas de los alumnos. Enseñanza de las Ciencias, 6(3), 285-290.

Vygotsky, L. (1995). Pensamiento y lenguaje. Barcelona: Paidós Ibérica.

White, R. T. y Gunstone, R. F. (1992). Probing understanding. Londres: The Falmer Press.

Worth, K. (2010). Science in early childhood classrooms: Content and process. Early Childhood Research and Practice, Collected Papers from the SEED (STEM in Early Education and Development) Conference, 10, 1-118.

Yin, R. K. (2003). Case study research. Design and methods. California: Sage Publications. 


\title{
Constructing concepts about electricity in early childhood education through inquiry-based activities
}

\author{
Nuria Calo Mosquera, Isabel García-Rodeja Gayoso,Vanessa Sesto Varela \\ Departamento de Didácticas Aplicadas, Universidade de Santiago de Compostela, \\ Santiago de Compostela, A Coruña, España \\ nuriacalotrazo@gmail.com, isabel.garcia-rodeja@usc.es, vanessa.sesto@usc.es
}

There is a growing consensus that science can be a particularly important domain in early childhood education. Some of the benefits of introducing science to noticeably young children are the construction of ideas that facilitate the understanding of scientific concepts that will be drawn upon more fully in later educational stages, the development of general skills linked to research design, and the generation of positive attitudes towards science. The topic of electricity and electrical circuits gives young children opportunities to reflect on ideas that are present in children's everyday life. This paper aims to describe the implementation of a teaching sequence where preschool children construct concepts and generalizations through empirical experience and dialogue with other when they engage in inquiry-based activities about electrical circuits.

The participants were seventeen children aged between 4 and 5 who took part in a teaching intervention about electrical circuits. This intervention was designed following a POE (Predict-Observe-Explain) strategy in which children are encouraged to make predictions about a phenomenon that they will then test through experimentation. The teaching intervention included three activities. During the first activity the teacher asked the children several questions about electricity to activate their ideas. During the second activity the children were given the opportunity to explore the elements of an electrical circuit. They also manipulated the circuits to turn up the light bulb. During the third activity the children were asked what would happen if a circuit were interrupted by placing different objects. This activity was aimed at promoting the construction of empirical references about the idea of conductive and non-conductive materials. Data collection included observation diaries and recordings of naturally occurring talk-in-interaction.

The findings point out that, in general, the children recognized that electricity is «something» that allows devices such as television or computer to work. They had no difficulty in identifying electrical and electronic devices. The idea that electricity is an entity generated by storms and windmills also emerge during children's interactions. However, children in this study hardly ever use the idea of electricity as something that flows through wires.

Regarding the evolution of ideas throughout the teaching intervention, we can point out that, at first, children explain their predictions and observations using ideas like the light bulb is not turned up because the object does not have electricity or is not well connected. However, as the children experiment with different objects, they begin to activate ideas related to the nature of the material from which the object is made. It is observed that children reach an inductive generalization according to which metals objects conduct electricity. Moreover, children show more difficulties in applying these ideas when they make predictions that when they justify their observations.

These findings suggest the need of conducting inquiry-based activities in early childhood education to give young children opportunities to develop thinking skills and generate empirical referents about scientific concepts. When designing teaching interventions, it is important to carry out prediction activities prior to the observation and give children the opportunity to justify both predictions and observations 